\title{
Observation of picosecond superfluorescent pulses in rubidium atomic vapor pumped by 100-fs laser pulses
}

\author{
Gombojav O. Ariunbold, ${ }^{1,2, *}$ Michael M. Kash, ${ }^{1,3}$ Vladimir A. Sautenkov, ${ }^{1,4}$ Hebin Li, ${ }^{1, \dagger}$ Yuri V. Rostovtsev, ${ }^{1,5}$ \\ George R. Welch, ${ }^{1}$ and Marlan O. Scully ${ }^{1,6,7}$ \\ ${ }^{1}$ Institute for Quantum Studies and Department of Physics, Texas A\&M University, College Station, Texas 77843, USA \\ ${ }^{2}$ Department of Physics, National University of Mongolia, 210646 Ulaanbaatar, Mongolia \\ ${ }^{3}$ Department of Physics, Lake Forest College, Lake Forest, Illinois 60045, USA \\ ${ }^{4}$ Lebedev Institute of Physics, Moscow 119991, Russia \\ ${ }^{5}$ Department of Physics, University of North Texas, 1155 Union Circle \# 311427, Denton, Texas 76203, USA \\ ${ }^{6}$ Applied Physics and Materials Science Group, Princeton University, Princeton, New Jersey 08544, USA \\ ${ }^{7}$ Max-Planck-Institute für Quantenoptik, D-85748 Garching, Germany
}

(Received 21 May 2010; published 20 October 2010)

\begin{abstract}
We study the superfluorescence (SF) from a gas of rubidium atoms. The atoms of a dense vapor are excited to the $5 D$ state from the $5 S$ state by a two-photon process driven by 100 -fs laser pulses. The atoms decay to the $6 P$ state and then to the $5 S$ state. The SF emission at $420 \mathrm{~nm}$ on the $6 P-5 S$ transition is recorded by a streak camera with picosecond time resolution. The time duration of the generated SF is tens of picoseconds, which is much shorter than the time scale of the usual relaxation processes, including spontaneous emission and atomic coherence dephasing. The dependence of the time delay between the reference input pulse and SF is measured as a function of laser power. The experimental data are described quantitatively by a simulation based on the semiclassical atom-field interaction theory. The observed change in scaling laws for the peak intensity and delay time can be elucidated by an SF theory in which the sample length is larger than the cooperation length.
\end{abstract}

DOI: 10.1103/PhysRevA.82.043421

PACS number(s): 42.50.Md, 42.50.Nn

\section{INTRODUCTION}

Spontaneous coherent emission from a collection of excited two-level atoms results in a burst of radiation. The peak intensity of this radiation is proportional to the square of the number of excited atoms in the medium. Dicke performed the first calculation of coherence in spontaneous radiation processes in 1954 [1] and he described this cooperative process as a "radiation bomb." In recognition of this groundbreaking work, this phenomenon is now referred to as Dicke superradiance, which is induced by a macroscopic dipole moment set up in the medium. Such radiation, however, can also occur even when an optically thick medium is prepared with a sufficiently large population inversion so that, eventually, the medium develops a macroscopic dipole moment resulting in superradiant emission [2]. In general, this is called superfluorescence (SF) [3].

An experimental realization of SF was pioneered by Skribanowitz et al. [4] using optically pumped hydrogen fluoride gas. The first observation of SF in rubidium vapor was reported by Marek [5].

Quantum and semiclassical theories of SF were initiated by Arecchi and Courtens [6], Rehler and Eberly [2], Bonifacio and Lugiato [3], and MacGillivray and Feld [7]. In the framework of the semiclassical approach, it was shown that the Maxwell-Bloch equations for the atomic system can be properly derived using quantum mechanics and only two parameters: an initial tipping angle $\theta_{0}$ and superradiant lifetime $\tau_{R}$ are adequate to determine the whole superradiant process [7]. The superradiant lifetime $\tau_{R}$ is approximately the average

\footnotetext{
*Corresponding author: ariunbold@ physics.tamu.edu

†Present address: JILA, University of Colorado and National Institute of Standards and Technology, Boulder, CO 80309-0440.
}

time for the first photon to be emitted along the input beam axis within the sample [8]. For the cylindrical sample, it is defined as [9]

$$
\tau_{R}=\frac{A T_{1}}{\mu N \lambda^{2}} .
$$

Here $T_{1}, \lambda, N, A$, and $\mu=3 / 8 \pi$ are the population decay time, the SF center wavelength, the number of the excited atoms, the sample area, and a geometrical factor [2], respectively.

One of the intriguing features of SF is a coherent ringing, first studied by Burnham and Chiao [10]. The so-called transverse effects for the ringing in SF were experimentally studied by Mattar et al. [11] and Heinzen et al. [12]. Good reviews of SF studies can be found in $[13,14]$.

SF involving cascade three-level atoms is called cooperative cascade emission. Both the theory and related experiment were first reported by Okada et al. [15]. The time development of cooperative cascade emission was also studied by using a streak camera with about 10 ps resolution [16]. A crucial example of cascade cooperative emission, the so-called yoked SF, was first observed in cesium vapor by Brownell et al. [17]. The experimental observation and theoretical description of the yoked SF with omnidirectional character (in rubidium vapor) were accomplished by Lvovsky et al. [18,19]. Recently, Paradis et al. obtained striking results while observing the yoked SF in laser-cooled rubidium atoms [20].

Although SF has been extensively studied, to the best of our knowledge no one has yet reported the generation of SF pulses several picoseconds in duration in an atomic vapor. This can be achieved if a dense gas of atoms is excited by laser pulses that are considerably shorter than the SF evolution time.

In this work, we use a rubidium vapor in which the atoms are excited into the $5 D$ state from the $5 S$ state by a two-photon 
process, which is enhanced by the $5 P$ intermediate level. This transition is driven by laser pulses of $100 \mathrm{fs}$ duration at $778 \mathrm{~nm}$. Many of the excited atoms decay into the $6 P$ state by emitting infrared light at $5 \mu \mathrm{m}$. SF emission occurs on the $6 P-5 S$ transition at $420 \mathrm{~nm}$, which is recorded by a streak camera with $\sim 2$ ps time resolution. The observed SF pulse duration is tens of picoseconds.

The same atomic configuration and associated radiation processes were recently considered by Lvovsky et al. [18,19] and Paradis et al. [20]. The delay between the input pulse and SF as a function of the number density of the atoms was presented by Lvovsky [19] by varying input pulse energy or the temperature of the vapor, and by Paradis [20] by loading different numbers of atoms into the magnetooptical trap.

In our work, we follow the former approach. The initial population of the upper level (the $5 D$ state) is varied by adjusting the laser input power. For relatively high laser powers, one can observe saturation and Rabi oscillations in both the $5 \mu \mathrm{m}$ and $420 \mathrm{~nm}$ emissions [19]. However, for low powers, far below the onset of saturation and/or oscillation, a simple correspondence between the input power and number of the excited atoms can be established. In this regime, we study the dependence of the time delay between the reference pulse and SF as the input beam power is varied, effectively changing the number of participating atoms.

The paper is organized as follows. In Sec. II, the experimental setup and measurement method are presented. In Secs. III and IV, the experimental and numerical results are listed. A comparison between the numerical and experimental results is also given in Sec. IV. Related discussions based on existing theories are given in detail in Sec. V. Section VI concludes the paper.

\section{EXPERIMENTAL DETAILS}

The atoms are initially prepared into a coherent superposition of the excited level $5 D$ and ground level $5 S$. Eventually, atoms decay to the ground state through the intermediate state $6 P$. The wavelengths of the generated fields in the upper, $5 D-6 P$, and lower, $6 P-5 S$, transitions are at $5 \mu \mathrm{m}$ and $420 \mathrm{~nm}$, respectively (see Fig. 1).

A schematic of our experiment is shown in Fig. 2. The input light was furnished by a commercial femtosecond laser system. The pulses entered a rubidium vapor cell. The generated pulses were analyzed by a spectrometer and a streak camera after passing through a colored absorption filter which is transparent for the $420 \mathrm{~nm}$ beam and blocks the $778 \mathrm{~nm}$ beam. Details of the experiment appear in the following.

The $778 \mathrm{~nm}$ (center wavelength) laser pulses were generated by an optical parametric amplifier (OPA) driven by a commercial femtosecond laser system. The resulting $778 \mathrm{~nm}$ pulses with repetition rate of $1 \mathrm{kHz}$ were vertically polarized with respect to the laser table and had a duration of $\sim 100 \mathrm{fs}$ as measured by a commercial autocorrelator. The beam was focused by a $20 \mathrm{~cm}$ plano-convex BK7 glass lens. The beam spatial profile at the focus was somewhat elliptical: full width at half maximum (FWHM) was $68 \mu \mathrm{m}$ (vertical) by $82 \mu \mathrm{m}$ (horizontal) as measured by the knife-edge method. Next the laser beam entered a rubidium vapor cell [21]. The cell was

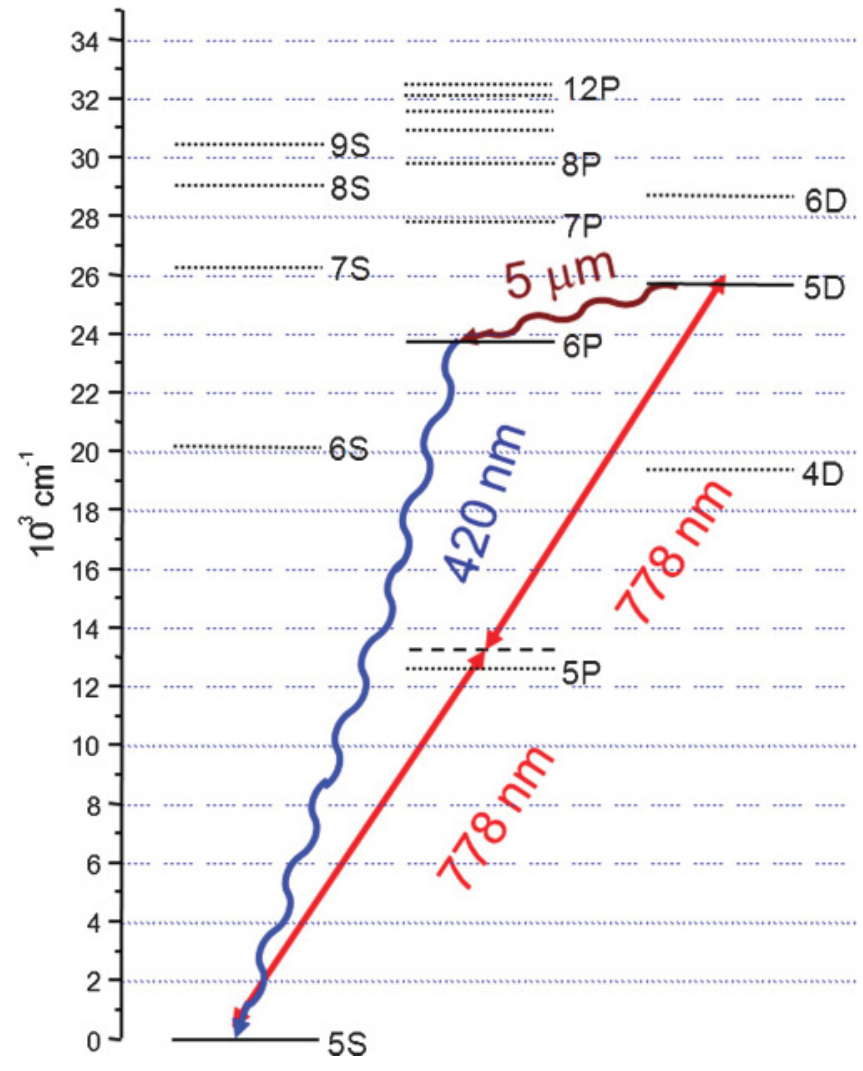

FIG. 1. (Color online) The rubidium atomic level scheme. After essentially instantaneous two-photon excitation of the input $778 \mathrm{~nm}$ pulse from the ground state $(5 S)$ to the upper level $(5 D)$, the atoms emit SF light at $5 \mu \mathrm{m}$ which triggers SF at $420 \mathrm{~nm}$.

fabricated from a sapphire body and garnet windows, and had a cylindrical shape with a length of $7 \mathrm{~cm}$ and a diameter of $1 \mathrm{~cm}$. A narrow tube was attached to the bottom of the cell, which is called the cold spot. The cell permitted high temperature operation and, except for the windows, was heated uniformly. To avoid atomic condensation, the windows were kept a bit hotter than the body of the cell. The data displayed in this paper were taken at the cold-spot temperature of $204^{\circ} \mathrm{C}$, as measured by a thermocouple, giving a rubidium density of $\sim 9 \times 10^{14} \mathrm{~cm}^{-3}$. The beam at $778 \mathrm{~nm}$ passed through a beam splitter and provided a time reference pulse for a streak camera. The reference beam passed through a corner-cube retroreflector mounted on a translation stage that provided an

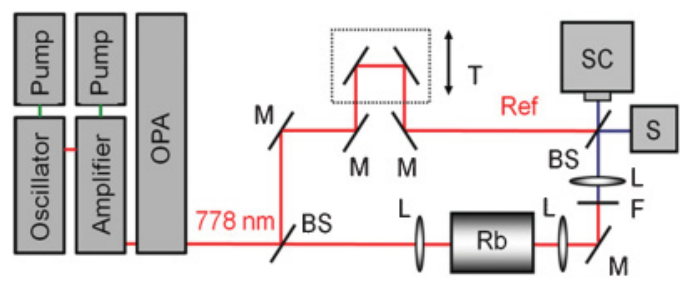

FIG. 2. (Color online) Diagram of experimental setup. OPA: optical parametric amplifier, $778 \mathrm{~nm}$ : input laser beam, BS: beam splitter, T: translation stage, M: mirror, L: lens, F: filter which is transparent for the $420 \mathrm{~nm}$ beam and blocks the $778 \mathrm{~nm}$ beam, SC: streak camera, S: spectrometer, Ref: timing reference beam, $\mathrm{Rb}$ : rubidium vapor cell. 
adjustable time delay. A second beam splitter combined the light from the cell with the time reference beam before both entered the streak camera (Hamamatsu). The violet light at $420 \mathrm{~nm}$ generated in the rubidium vapor was emitted primarily in a narrow beam, parallel to the laser beams. The $420 \mathrm{~nm}$ beam had a divergence of about $8 \mathrm{mrad}$. Upon exiting the cell, the beam was collimated and made parallel by a lens with a $10 \mathrm{~cm}$ focal length. The $778 \mathrm{~nm}$ beam was isolated by a filter. This filter was transparent to the $420 \mathrm{~nm}$ beam. The $420 \mathrm{~nm}$ light was focused by another lens (focal $15 \mathrm{~cm}$ ) into the streak camera's entrance slit. The beam splitter reflected some $420 \mathrm{~nm}$ light toward the input fiber of the spectrometer. The power of the input and reference beams were adjusted by two variable neutral density filters, which are not shown in Fig. 2.

\section{EXPERIMENTAL RESULTS}

We first recorded the spectra of the $420 \mathrm{~nm}$ light for different input powers. In Fig. 3 the magnitude at $420 \mathrm{~nm}$ light from the measured spectra is plotted as a function of input power, and shows a monotonic dependence. However, for sufficiently high power the dependence becomes much more complex [19].

Next, to detect the temporal characteristics of the generated signal, we used the fast streak camera. We concentrated on the measurement of the average time delay. The main results are shown in Fig. 4. The $420 \mathrm{~nm}$ light and reference beam were recorded by the streak camera. The set of measurements was done for several input powers ranging from 0.3 to $2.1 \mathrm{~mW}$ (note that $1 \mathrm{~mW}$ average power corresponds to $\sim 1 \mu \mathrm{J} /$ pulse). Because the signal was not stable, mostly due to laser jitter, up to 100 shots were averaged after jitter correction. This correction procedure results in an additional systematic error in time resolution. The time duration of the reference pulse was about $100 \mathrm{fs}$, which should ideally be displayed by streak camera as an $\sim 2$ ps pulse, the limit of its resolution. In our case, the FWHM of the reference signal was approximately $\sim 6$ ps ( $>2$ ps).

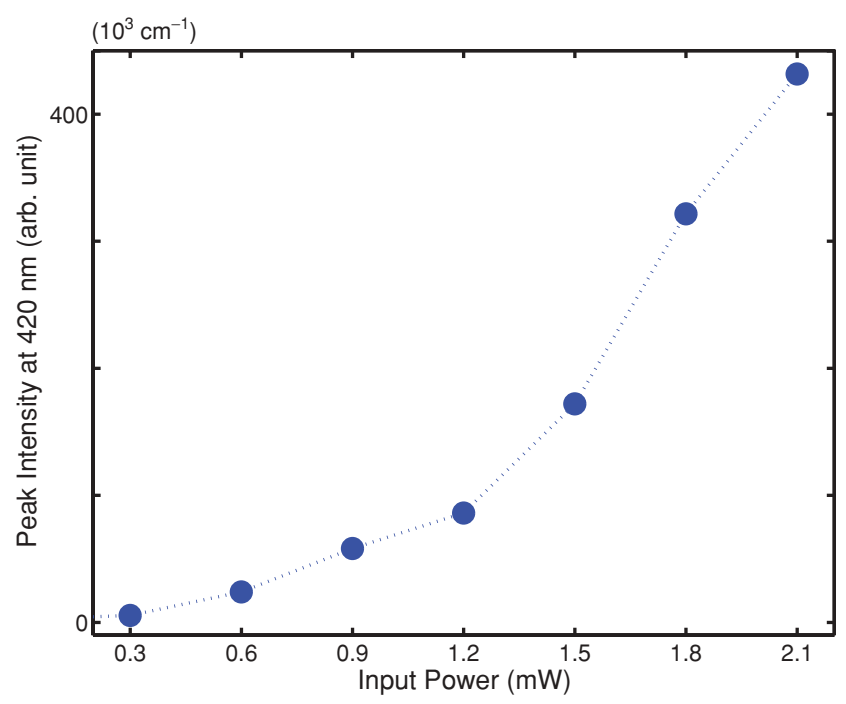

FIG. 3. (Color online) The power dependence of the magnitude at $420 \mathrm{~nm}$ from the recorded spectra.

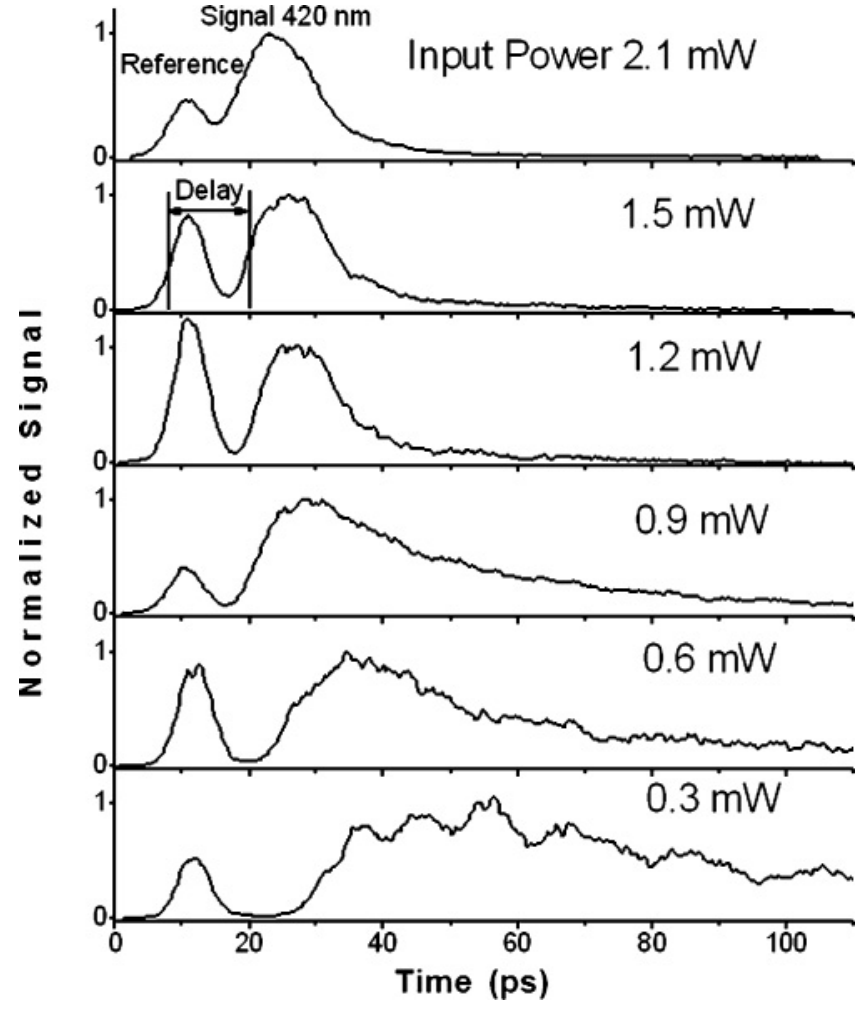

FIG. 4. The streak camera data for the reference and $420 \mathrm{~nm}$ pulses. The input beam power was varied from 0.3 to $2.1 \mathrm{~mW}$.

One of the intriguing temporal properties of SF is ringing. The result for $\sim 0.3 \mathrm{~mW}$ input power clearly exhibited this feature. In Fig. 4, the $420 \mathrm{~nm}$ signal was normalized: Each measurement was taken with different gain settings of the camera and attenuation of the neutral density filters to improve the signal-to-noise ratio. As a result, in Fig. 4, the peaks of the reference beam have different heights. The peaks of the reference beams are adjusted to coincide with each other in time, thus enabling us to distinguish relative delays for each pulse. Generally, the temporal behavior of the $420 \mathrm{~nm}$ signal had a steep rising edge with moderately long tail. The delay is defined as a time interval between the reference and $420 \mathrm{~nm}$ pulses, as shown in Fig. 4. The delay was calculated at the half maximum of the rising edge of the reference and $420 \mathrm{~nm}$ pulses and summarized as a function of input power in Fig. 5. The error bars are estimated to be $\sim 3$ ps for the average delay and $\sim 0.05 \mathrm{~mW}$ for the average power measurements. It is obvious from the observed data that SF appears sooner as input pulse energy increases.

\section{NUMERICAL RESULTS}

The semiclassical theory is presented in this section. To include the pulse excitation explicitly, we consider a five-level atomic system. Figure 6 defines $|c\rangle$ as a ground level ( $5 S$ state), $|a\rangle$ as an upper level (5D state), $\left|b_{1}\right\rangle$ and $\left|b_{2}\right\rangle$ as the intermediate levels (two $D$ lines, $5 P_{1 / 2}$ and $5 P_{3 / 2}$ states, respectively) for two-photon excitation, and $|b\rangle$ as another intermediate level (6P state) for yoked-SF emissions. The frequency $v_{\alpha \beta}$ and the Rabi frequency $\Omega_{\alpha \beta}$ stand for the field mode coupled to the transition $|\alpha\rangle \leftrightarrow|\beta\rangle$. Note that the initial value of the Rabi 


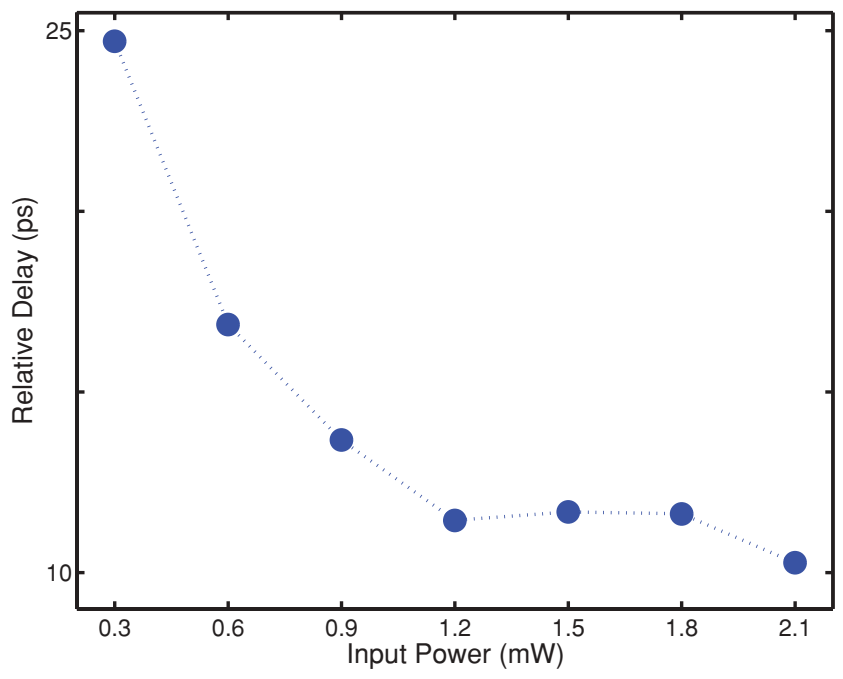

FIG. 5. (Color online) The relation between the relative time delay with respect to the arrival time of the reference pulse and input power.

frequency $\Omega_{\alpha \beta}$ is a very small phenomenological parameter, to start the temporal development of the system [17]. The two-photon detuning is $\Delta_{2}=\left(\omega_{a}-\omega_{c}\right)-2 v_{\text {in }}$, where $\omega_{i}$ is the frequency of the $i$ th level and $v_{\text {in }}$ is the frequency of the input field. In contrast, the single-photon detuning is $\Delta_{1}=v_{\text {in }}-\left(\omega_{b_{1}}-\omega_{c}\right)$. The splitting between $D$ lines is $\Delta_{0}=\omega_{b_{2}}-\omega_{b_{1}}$. One of the important parameters is the input field's Rabi frequency $\Omega_{\text {in }}$. In the semiclassical treatment, the interaction of the atom with the classical field is governed by the Maxwell-Bloch equations in a retarded time frame. The semiclassical Hamiltonian in the interaction picture for this system can be written as $(\hbar \equiv 1)$

$$
\begin{aligned}
\hat{V}(t)= & -\Omega_{\text {in }}\left(e^{-i \phi_{1} t}|a\rangle\left\langle b_{1}\left|+e^{-i \phi_{2} t}\right| a\right\rangle\left\langle b_{2}\left|+e^{-i \Delta_{1} t}\right| b_{1}\right\rangle\langle c|\right. \\
& \left.+e^{-i\left(\Delta_{1}-\Delta_{0}\right) t}\left|b_{2}\right\rangle\langle c|\right)-\Omega_{a b}|a\rangle\left\langle b\left|-\Omega_{b c}\right| b\right\rangle\langle c|+\text { H.c. },
\end{aligned}
$$

where $\phi_{1}=2 v_{\text {in }}-\left(v_{a b}+v_{b c}\right) \equiv-\Delta_{2}$ and $\phi_{2}=\phi_{1}+\Delta_{0}-$ $\Delta_{1}$. The equation of motion for the atomic density operator $\hat{\rho}$ is given by

$$
\frac{\partial \hat{\rho}}{\partial t}=-i[\hat{V}(t), \hat{\rho}] .
$$

Note that no population decay or atomic coherence dephasing is included here.

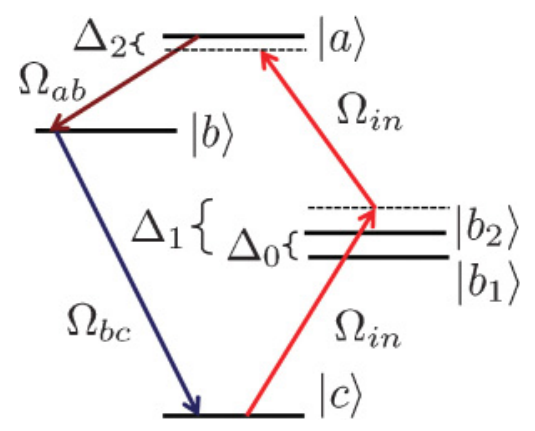

FIG. 6. (Color online) A model of a five-level atomic system, see text for detail.

\section{A. Yoked superfluorescence}

We consider the field generated in the axial direction, that is, along the $z$ axis. Assuming that the backward $5 \mu \mathrm{m} \mathrm{SF}$ is uncoupled from the forward $5 \mu \mathrm{m}$ and forward $420 \mathrm{~nm} \mathrm{SF}$, the Maxwell equations for two copropagating modes in the retarded time frame $t \rightarrow t-z / c$, are given by $[15,17,22]$

$$
\begin{aligned}
& \frac{\partial \Omega_{a b}}{\partial z}=i \eta_{a b} \rho_{a b} \\
& \frac{\partial \Omega_{b c}}{\partial z}=i \eta_{b c} \rho_{b c} .
\end{aligned}
$$

Here the Rabi frequency can be expressed in terms of the field amplitude $E_{\alpha \beta}$ and transition dipole moment $\wp_{\alpha \beta}$ as $\Omega_{\alpha \beta}=\wp_{\alpha \beta} E_{\alpha \beta}$. The relevant coupling constant $\eta_{\alpha \beta}$ is given by $2 \pi \mathcal{N}\left|\wp_{\alpha \beta}\right|^{2} \omega_{\alpha \beta} / c$, where $\mathcal{N}$ is the atomic number density, $\omega_{\alpha \beta}=\omega_{\alpha}-\omega_{\beta}$ is the transition frequency between the $\alpha$ th and $\beta$ th levels, and $c$ is the speed of light.

It is useful to simplify the present model and find analytical solutions because it furnishes a qualitative understanding of the observed experimental results. The input pulse duration used in the experiment is $100 \mathrm{fs}$, which is an instantaneous excitation compared to the delay and duration of the yoked-SF pulse. Thus the three-level atomic configuration should be a good approximation. In this case, the initial populations of the upper and lower states are the control parameters rather than input pump power. This model of copropagating modes for cascade three-level atoms has been studied in $[15,22]$ where the backward propagating mode is assumed to be generated independently. The reduced equation of motion for three-level atoms can be obtained from the five-level case by eliminating levels $\left|b_{1}\right\rangle$ and $\left|b_{2}\right\rangle$. For the sake of simplicity, assume that the populations of the levels are time independent and expressed by their initial values [i.e., $\rho_{c c}(t) \simeq \rho_{c c}(0), \rho_{a a}(t) \simeq \rho_{a a}(0)$, and $\rho_{b b}(t) \simeq 0$ ]. In the linear approximation [22], nonlinear terms $\Omega_{b c} \rho_{a b}$ and $\Omega_{a b} \rho_{b c}$ are neglected; thus, $\rho_{a c}(t) \simeq \rho_{a c}(0)$. Similarly, as found in $[15,17,22]$, the Rabi frequencies are given by

$$
\begin{gathered}
\Omega_{a b}(z, t) \propto-\eta_{a b} \rho_{a c}(0) I_{0}(2 \sqrt{\xi z t}), \\
\Omega_{b c}^{*}(z, t) \propto \eta_{b c} \rho_{c c}(0) I_{0}(2 \sqrt{\xi z t}),
\end{gathered}
$$

where $\xi=\eta_{a b} \rho_{a a}(0)-\eta_{b c} \rho_{c c}(0)$ and $\left|\rho_{a c}(0)\right|^{2}=\rho_{a a}(0) \rho_{c c}(0)$. From the approximate solutions in Eq. (4), we infer the characteristic exponential growth [22] of the emissions on both the upper $(a b)$ and lower $(b c)$ transitions (i.e., both the $5 \mu \mathrm{m}$ and $420 \mathrm{~nm}$ pulses are SF emissions [17]). The growth rate does depend solely on the main parameter $\xi$ leading to yoked $\mathrm{SF}$ [17]. In other words, there is no delay between the $5 \mu \mathrm{m}$ and $420 \mathrm{~nm}$ pulses. Equation (4) also shows that the passage time at which the field intensity reaches a specified value [23] depends on the initial population $\rho_{a a}(0)$ since $\eta_{a b}>\eta_{b c}$. The increase of the population leads to a faster growth (i.e., a shorter delay).

At the next stage, we numerically solve Eq. (2) together with Eq. (3). The normalized $5 \mu \mathrm{m}$ and $420 \mathrm{~nm}$ SF pulse shapes are shown in Fig. 7. The excitation input pulse is assumed to be a Gaussian with amplitude $\Omega_{0}=3$. The coupling parameters used here are $\eta_{a b}=4$ and $\eta_{b c}=0.08$, and the sample length is $L=160$. In addition, we assume that all initial populations are zero except for $\rho_{c c}(0)=1$. The other parameters are taken 


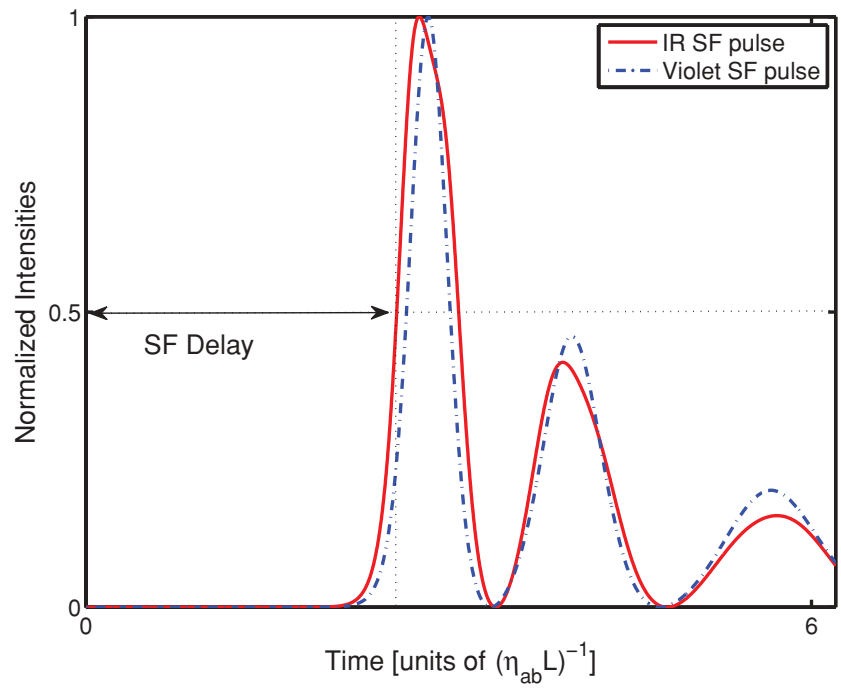

FIG. 7. (Color online) The time evolution of the normalized intensities $\left|\Omega_{a b}\right|^{2}\left(5 \mu \mathrm{m}\right.$ pulse) and $\left|\Omega_{b c}\right|^{2}$ (420 nm pulse) for an input Gaussian pulse with amplitude $\Omega_{0}=3$.

to be $\Delta_{0}=C(1 / 780-1 / 795)$ (splitting between rubidium $D$ lines), $\Delta_{1}=C(1 / 778-1 / 795)$ (detuning between rubidium $D_{1}$ line and the wavelength of the input pulse), where $C=7 /(1 / 780-1 / 795)$ and $\Delta_{2}=0$ (two-photon detuning). Here $\Delta_{0}, \Delta_{1}, \Delta_{2},\left(\eta_{a b} L\right)$, and $\left(\eta_{b c} L\right)$ have units of THz; time $t$ has units of ps. The SF delay can be calculated at the half maximum of the pulse of the rising edge (see Fig. 7). The delay varies when input pulse amplitude $\Omega_{0}$ is changed from 2.5 to 4.5 . The delay dependence on amplitude of the input Gaussian pulse is plotted in Fig. 8. As is seen from Fig. 8, the forward $5 \mu \mathrm{m}$ and $420 \mathrm{~nm} \mathrm{SF}$ pulses are developed simultaneously. While these numerical results provide qualitative agreement with the experimental data, they do not, however, provide quantitative agreement. This is because we assume that the forward and backward SF

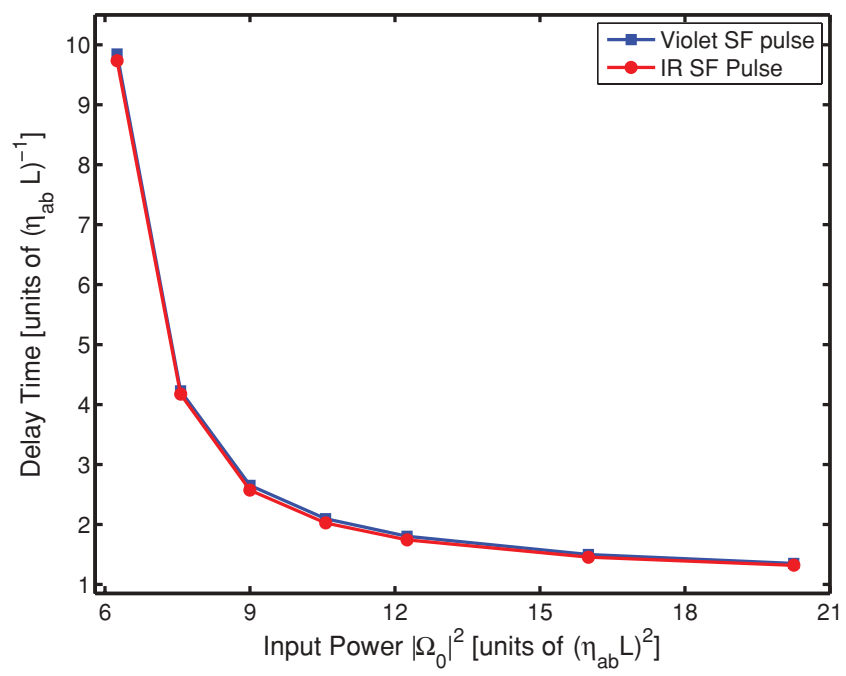

FIG. 8. (Color online) The simulations for delay times of the $5 \mu \mathrm{m}$ and $420 \mathrm{~nm}$ pulses as functions of the amplitude of the input Gaussian pulse. The parameters are the same as used in Fig. 7, but $\Omega_{0}=2.5,2.75,3,3.25,3.5,4$, and 4.5 . pulses are developed independently. In the following section, we simulate the backward propagating SF pulse.

\section{B. Phenomenological theory for backward superfluorescence and its connection to experiment}

The coupling between the backward and forward SF for two-level atoms has been studied in [24]. Recently, Brownell et al. [17] have observed a cascade SF in the backward direction. Inclusion of all SF pulses simultaneously in the general Maxwell equations is cumbersome. In the previous section it is assumed that the SF emissions in the forward and backward directions are independent [17,22] (i.e., the backward $5 \mu \mathrm{m} \mathrm{SF}$ is uncoupled from the forward $5 \mu \mathrm{m}$ $\mathrm{SF}$ and forward $420 \mathrm{~nm} \mathrm{SF}$ ). In contrast, let us assume here that the backward $5 \mu \mathrm{m}$ SF and forward $420 \mathrm{~nm}$ SF are coupled, and the forward $5 \mu \mathrm{m} \mathrm{SF}$ is independent. Further, we make the following assumption. The propagation equation for the backward $5 \mu \mathrm{m} \mathrm{SF}$ is written in time frame $t-z / c$, while the equation for the forward $420 \mathrm{~nm} \mathrm{SF}$ is written in time frame $t+z / c$. Thus, the phenomenological propagation equations for two modes traveling in opposite directions are given by

$$
\begin{gathered}
\frac{\partial \Omega_{a b}}{\partial z}=i \eta_{a b} \rho_{a b}, \\
-\frac{\partial \Omega_{b c}}{\partial z}=i \eta_{b c} \rho_{b c} .
\end{gathered}
$$

Here, the $z$ axis is taken to be along the backward $5 \mu \mathrm{m}$ light propagation direction. The $420 \mathrm{~nm}$ SF pulse shapes are shown in Fig. 9 for several different input amplitudes. All other parameters are the same as used in Fig. 7. The curves in Fig. 9 are similar to ones in Fig. 4. In Fig. 9, if the square of the Rabi frequency $\left|\Omega_{0}\right|^{2}$ is scaled by some factor $\alpha$, then time should be scaled by $\sqrt{1 / \alpha}$. Comparing the pulse temporal shapes for $\left|\Omega_{0}\right|^{2}=6.25$ and 25 with the experimental results for input powers $0.3 \mathrm{~mW}$ and $1.2 \mathrm{~mW}$, we choose $\alpha=0.0480$. We are now able to quantitatively match the simulation to the experimental data. Note that, in the experiment, the delay between the input reference (but not the pump) and

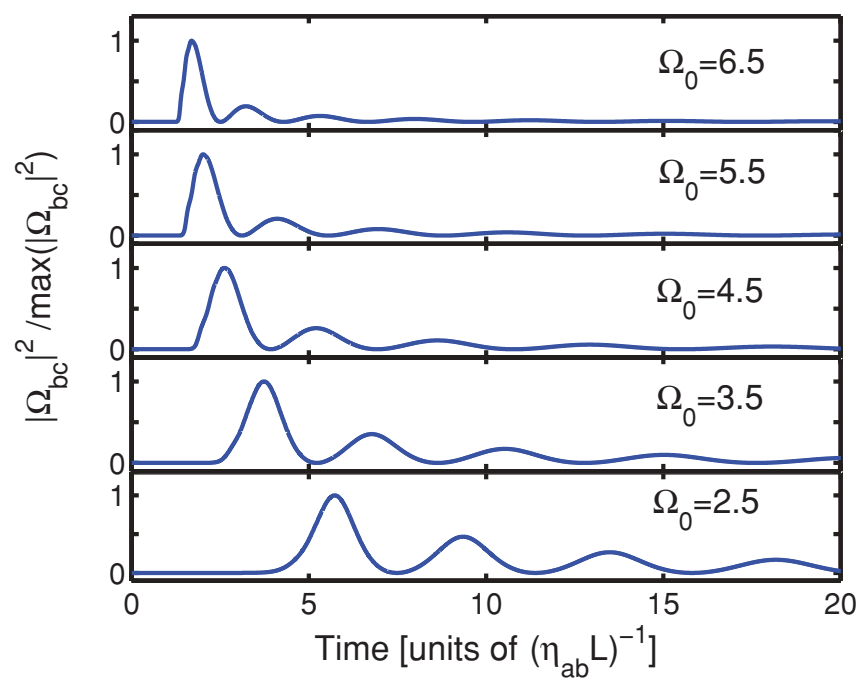

FIG. 9. (Color online) The time evolution of the normalized intensity $\left|\Omega_{b c}\right|^{2}$ for different input pulse amplitudes. The input Gaussian pulse amplitudes are $\Omega_{0}=2.5,3.5,4.5,5.5$, and 6.5. 


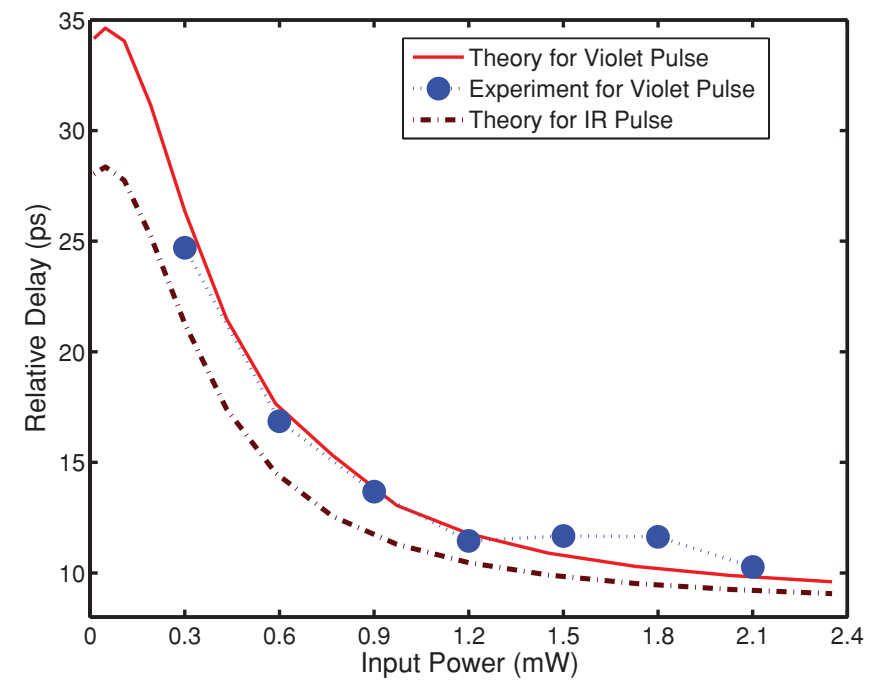

FIG. 10. (Color online) The measured relative delay time for the $420 \mathrm{~nm}$ pulse as a function of input power (full circles) and simulations for both the $5 \mu \mathrm{m}$ (dotted curve) and $420 \mathrm{~nm}$ (solid curve) pulses with scaling factor $\alpha=0.0480$.

$420 \mathrm{~nm}$ pulse was measured. Because of the additional optical pathlengths resulting from the lens, beam splitter, and filters used in the detection configuration, an accurate time delay with respect to the pump cannot be measured directly. However, in a special case, there is a certain technique to measure this delay accurately using the higher-order harmonics of the input laser pulses [25]. It is important to know the delay accurately as this knowledge helps us to understand the details of the experiment, such as the number of atoms participating in the cooperative emission. This particular example will be discussed in Sec. V.

Keeping in mind the fact that the simulated delay time is relative to the pump, we find the offset to be $\sim 3.2 \mathrm{ps}$. The simulated (solid curve) and experimental (full circles) results for delay times of the $420 \mathrm{~nm}$ pulse are shown in Fig. 10. An excellent agreement between the experiment and simulation is obtained. It must be borne in mind that there is no free fitting parameter used in the simulations. We simply start with the semiclassical Hamiltonian and numerically solve Eqs. (2) and (5). The streak camera, lens, and filters we used in the experiment are not appropriate for detection of the $5 \mu \mathrm{m}$ pulse. Lvovsky et al. [19], have obtained partial information for the $5 \mu \mathrm{m}$ light using a germanium detector. In this paper, the results obtained for the $420 \mathrm{~nm}$ pulse reasonably predict the delay time dependence for the $5 \mu \mathrm{m}$ pulse. The dotted curve in Fig. 10 stands for the $5 \mu \mathrm{m}$ pulse delay dependence on input power; it increases as power decreases. A nonzero delay occurs between the generations of the $5 \mu \mathrm{m} \mathrm{SF}$ and $420 \mathrm{~nm}$ SF. Brownell et al. [17] found that the backward SF pulse in the upper transition arrived first in time, followed by the yoked-SF emissions in both the upper and lower transitions in the forward direction. In this sense, one should anticipate some delay between the backward $5 \mu \mathrm{m} \mathrm{SF}$ and forward $420 \mathrm{~nm}$ SF for both the cascade and yoked SF in the forward direction; unfortunately, we could not give a reasonable physical explanation for this phenomenon. To do that one needs a rigorous analytical treatment of the cascade three-level atomic system, which would be beyond the framework of this paper.

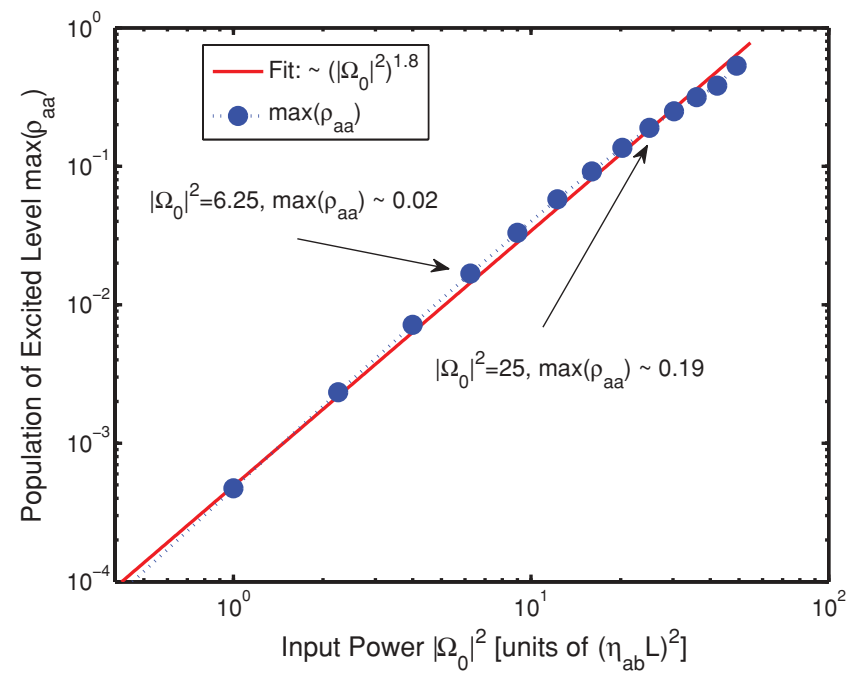

FIG. 11. (Color online) The log-log scaled relations of the population of the excited state $\max \left(\rho_{a a}\right)$ (full circles) and its fit with function $\left|\Omega_{0}\right|^{3.6}$ (solid curve) and input intensity.

Since the experiment and simulation are in agreement for the $420 \mathrm{~nm}$ violet pulses, we can find the delay relative to the pump. For instance, at 1.2 and $0.3 \mathrm{~mW}$ input power, the relative delay is found to be 8.6 and $23.2 \mathrm{ps}$, respectively. Similarly, for the $5 \mu \mathrm{m}$ pulses at 1.2 and $0.3 \mathrm{~mW}$ input power, these are 7.3 and $18.1 \mathrm{ps}$, respectively. So far, the delay times have been determined at the rising edge of the pulse. The $5 \mu \mathrm{m}$ pulse delay defined at its maximum is given by $19.6 \mathrm{ps}$ at $0.3 \mathrm{~mW}$ (7.6 ps at $1.2 \mathrm{~mW})$. Note that in the recent work [25], we probe the excitation of the $5 D$ state using an extra ir input pulses. The delayed ir pulse probes the population of the $5 D$ state to show that it decays in $\sim 16$ ps which is consistent with the results obtained here.

The population of the excited state is calculated numerically as the amplitude of the input pulse varies. In Fig. 11, the population of the excited state $|a\rangle$ (full circles) is plotted as a function of the input Gaussian pulse intensity. A fit (solid curve) is given by the formula $\left|\Omega_{0}\right|^{2 x} / 10^{7.6}$ with $x=1.8$. The population is expected to be proportional to the square of input energy, as is typical for the two-photon transient excitation. Using the scaling factor $\alpha=0.0480$, we can estimate the population of the excited state $\rho_{a a}$ as a function of input power. In Fig. 11 it is shown that for particular input values $\left|\Omega_{0}\right|^{2}=6.25$ and 25 , the populations are $\sim 0.02$ and $\sim 0.19$, respectively. This means that at 0.3 and $1.2 \mathrm{~mW}$ input powers only $2 \%$ and $19 \%$ of the atoms are excited, respectively. Fifty percent of the atoms can be excited at $2.1 \mathrm{~mW}$. A simple relation between population and input intensity supports the assumptions for the three-level atomic model.

\section{DISCUSSIONS BASED ON EXISTING THEORIES}

In this section, we discuss the main results in terms of existing theories. Let us estimate the number of atoms in the focal region. The diameter of the focused beam at the focus of the lens is $d=4 \lambda_{778 \mathrm{~nm}} f /(\pi D) \sim 80 \mu \mathrm{m}$, where the unfocused beam diameter is $D=2.5 \mathrm{~mm}$, and lens focal length is $f=20 \mathrm{~cm}$. This is consistent with the measured beam sizes at the focal plane, which are 68 and $82 \mu \mathrm{m}$. 
The depth of focus $\mathrm{D}_{\mathrm{OF}}=8 \lambda_{778 \mathrm{~nm}} f^{2} /\left(\pi D^{2}\right) \sim 1 \mathrm{~cm}$. Thus, at a temperature of $204^{\circ} \mathrm{C}$, the total number of atoms in this volume is $N_{\text {total }}=\pi d^{2} / 4 \mathrm{D}_{\mathrm{OF}} \mathcal{N} \sim 4.5 \times 10^{10}$ where the number density $\mathcal{N}$ is $9 \times 10^{14}$, and the area is $\pi d^{2} / 4$.

It was first mentioned by Arecchi and Courtens in 1971 [6] that there is a restriction to the number of cooperative atoms due to the fact that the photons travel with finite speed. For instance, if the sample length is longer than the product of SF delay and the speed of light, then the remote atoms cannot be considered as cooperative. This restriction is critical in our case since the SF pulse delay and width are measured in tens of picoseconds.

Let us first compare the delays at the specific powers, 0.3 and $1.2 \mathrm{~mW}$. Earlier, we found that at 0.3 and $1.2 \mathrm{~mW}$ the $5 \mu \mathrm{m}$ SF pulse has 19.6 and 7.6 ps delays (defined at the maximum of the pulse), respectively. The corresponding cooperation lengths are $l_{0.3 \mathrm{~mW}} \sim 5.9 \mathrm{~mm}$ and $l_{1.2 \mathrm{~mW}} \sim 2.3 \mathrm{~mm}$. At this stage, we can estimate the Fresnel number $F_{5 \mu \mathrm{m}}=$ $2\left(\pi d^{2} / 4\right) /\left(L \lambda_{5 \mu \mathrm{m}}\right)$, which was used by MacGillivray and Feld [7], as $1 \leqslant F_{5 \mu \mathrm{m}} \leqslant 3$. The maximum cooperation number is given by $N=A l \mathcal{N}$. Taking into account the percentage of the excitation for two powers, the number of excited atoms are estimated to be

$$
\begin{aligned}
& N_{0.3 \mathrm{~mW}}=0.02 A l_{0.3 \mathrm{~mW}} \mathcal{N}=5 \times 10^{8} \\
& N_{1.2 \mathrm{~mW}}=0.19 A l_{1.2 \mathrm{~mW}} \mathcal{N}=2 \times 10^{9} .
\end{aligned}
$$

The ratio of these values is $N_{1.2} \mathrm{mw} / N_{0.3 \mathrm{~mW}} \sim 4$, whereas the ratio for delay times is $19.6 \mathrm{ps} / 7.6 \mathrm{ps} \sim 3$. Therefore, based on the above estimation, the emission on the upper transition ( $5 \mu \mathrm{m}$ pulse) is SF in which the inverse of the delay is approximately proportional to the number of the excited cooperative atoms.

According to Polder et al. [26], the ratio between delay time $\tau_{D}$ and the superradiant lifetime $\tau_{R}$ is

$$
\frac{\tau_{D}}{\tau_{R}}=\frac{1}{4}\left[\ln (2 \pi N)^{1 / 2}\right]^{2} .
$$

In particular, for $N_{1.2 \mathrm{~mW}}=2 \times 10^{9}$, this ratio is given by $\tau_{D} / \tau_{R} \sim 34$. From Eq. (1) the superradiant lifetime is $\tau_{R} \sim$ $0.6 \mathrm{ps}$ for $T_{1}=0.69 \mu \mathrm{s}$ and $\lambda=5 \mu \mathrm{m}$. Therefore, delay time becomes $\tau_{D} \sim 20$ ps. For $N_{0.3 \mathrm{~mW}}=5 \times 10^{8}$ the ratio is $\tau_{D} / \tau_{R} \sim 30$. The delay time is $\tau_{D} \sim 69 \mathrm{ps}$ for $\tau_{R} \sim 2.3 \mathrm{ps}$. The experiment suggests that delay times for $1.2 \mathrm{~mW}$ and $0.3 \mathrm{~mW}$ are $7.6 \mathrm{ps}$ and $19.6 \mathrm{ps}$, respectively. According to the above estimation, we found 20 and 69 ps. These values are off by a factor of $\sim 3$. This may be corrected with a different geometrical factor. In any case, the ratio between delays in theory and experiment are $69 \mathrm{ps} / 20 \mathrm{ps} \sim 3$ and $19.6 \mathrm{ps} / 7.6 \mathrm{ps} \sim 3$, respectively, yielding a consistent result.

Next we study the changes in scaling laws for the peak intensity and delay time in more detail. To be more precise, let us consider the experimental data for powers from 0.3 to $1.2 \mathrm{~mW}$. In Fig. 12 we plot the experimental data for the relationship between peak intensity and input power. A fit (solid curve) of the data (full circles) demonstrates that the peak intensity of the $420 \mathrm{~nm}$ light is approximately proportional to the square of input power as $I_{\max } \propto P^{x}$, where $x=2.0+\Delta x$. Taking into account the experimental errors in

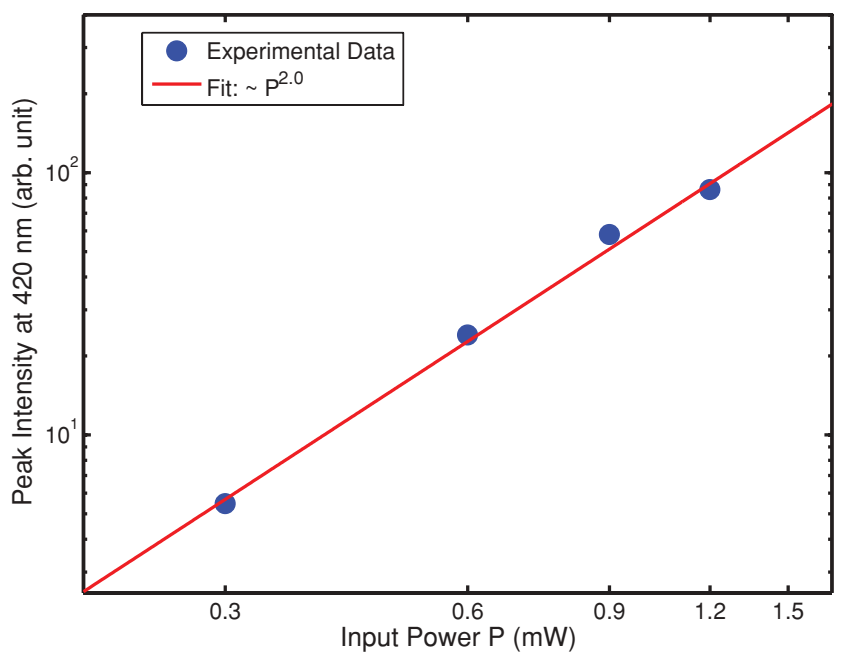

FIG. 12. (Color online) The power dependence of the magnitude at $420 \mathrm{~nm}$ from the recorded spectra for the $420 \mathrm{~nm}$ SF light (full circles) and its fit with function $P^{2.0}$ (solid line) in a log-log scale.

power measurement we find $-0.2<\Delta x<0.2$. For the case of the two-photon pumping, the number of participating atoms is proportional to the square of input power as $N \propto P^{2}$ (see Fig. 11). This means that the peak intensity is proportional to $N$, but not $N^{2}$. In Fig. 13, we show the experimental data (full circles) for the relative delay versus input power in a $\log -\log$ scale. In this figure, the dashed line and dotted line correspond to the power laws $P^{-1}$ and $P^{-2}$, respectively. We fit the delay data to the form $a / P^{x}+b$, where a fitting parameter $x$ provides information about the power law. In Sec. IV, we estimated the temporal offset to be $b=3.2 \mathrm{ps}$. Using this offset, from the fit (solid curve) we obtain $x=0.7+\Delta x$. In view of the error bars in the delay and power measurements, $\Delta x$ is found to be $-0.2<\Delta x<0.3$. For pure SF [3], the peak intensity and time delay scale as $N^{2}$ and $N^{-1}$, respectively. For the oscillatory SF [13,27], they are scaled by $N$ and $N^{-1 / 2}$. Therefore, our observed data can be explained best by the

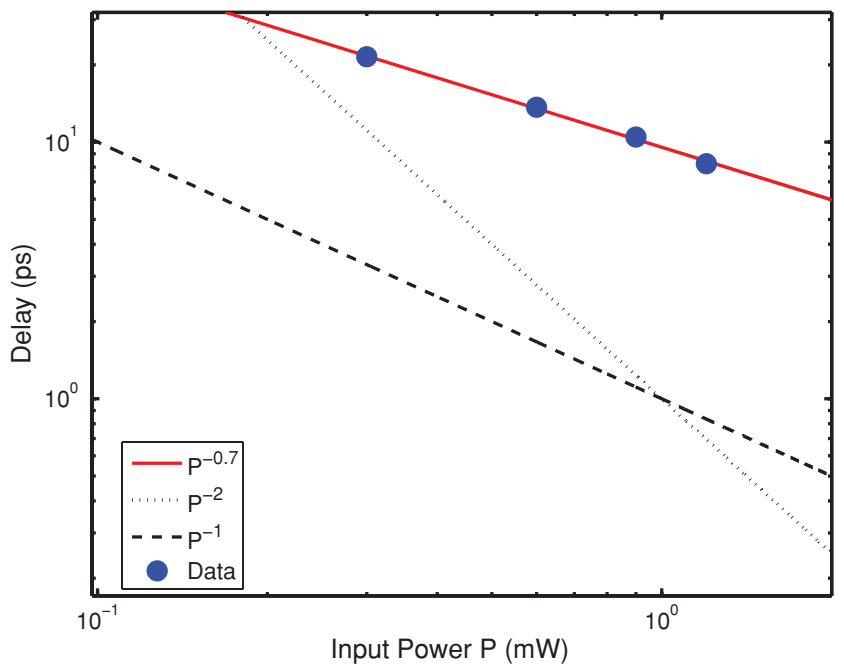

FIG. 13. (Color online) A log-log plot that shows the $420 \mathrm{~nm} \mathrm{SF}$ delay time relative to the reference pulse as a function of input power (full circles). A fit is given by $\propto P^{-0.7}$ (solid line). The data are also compared to functions $P^{-2}$ (dotted line) and $P^{-1}$ (dashed line). 
oscillatory SF theory. A little discrepancy between the fit $N^{-x / 2}$ and prediction for the oscillatory SF, $N^{-1 / 2}$, can be explained by the following. (i) The data for the delay were measured at the rising edge but not at the peak of the SF pulse. (ii) The data were taken for the SF at $420 \mathrm{~nm}$ rather than the SF at $5 \mu \mathrm{m}$. A delay between the $5 \mu \mathrm{m}$ SF and $420 \mathrm{~nm} \mathrm{SF}$ is nonzero. Considering these points one would obtain more precise agreement. Nevertheless, even without the above-mentioned points, we can clearly recognize the consistency of the data with $N^{-1 / 2}$ dependence.

Finally we discuss the temporal ringing in more detail. The SF temporal ringing in two-level atoms has been widely studied [7,10-12,27-29]. However, the ringing has not been investigated in a cascade three-level system. The detailed theory for temporal ringing in this case would be much more complicated because of the cascade emissions. It can be simplified under the yoked-SF condition where the SF emissions on the upper and lower transitions occur simultaneously. In this case, one can consider the yoked SF on the lower transition as the same as that on the upper transition (i.e., the SF for two-level atoms).

The origin of temporal ringing is twofold. The MaxwellBloch theory predicts an intrinsic SF ringing [7]. In this case, the temporal ringing results from the energy exchange between field and atoms while the field is propagating inside a single zone (slice) [27]. On the other hand, the ringing occurs when the field is reabsorbed and reemitted while propagating through multiple cooperation zones [27,29]. We did not perform a specific experiment to distinguish the two types of ringing, and in the present situation it could possibly be a mixture of the two. However, in Fig. 4, the temporal ringing is caused by the energy exchange between field and atoms within a single cooperative zone. From Fig. 4, one can estimate that the width is less than $5 \mathrm{ps}$ (the width of the SF pulse at $0.3 \mathrm{~mW}$ ), and the delay is longer than $15 \mathrm{ps}$ (a time interval between arrival times of the SF pulses for low and high power). Their ratio is greater than 3. According to [27], if the ratio is much greater than unity then an intrinsic SF ringing is important. It is also interesting to note that the observed temporal ringing was reproducible. The temporal ringing demonstrated a quasiperiodic dependence on the square root of time variable. Therefore the ringing was not due to a succession of SF pulses from multiple cooperation zones with diminishing intensity [29].

Both the diffraction and transverse effects can cause the reduction of the ringing [13]. The diffraction effect is negligible in this case because the Fresnel number is greater than 1 for a single zone even if it is close to 1 for the entire sample. The transverse effect is expected to be dominant since the input light was focused into the sample. The transversal plane of the input beam leads to a nonuniform excitation of the atoms and the emissions smear out the ringing. In particular, when input power increases, the length of the cooperative zone decreases (i.e., the emissions from the atoms on the transversal plane would be more noticeable).
According to simulations based on the semiclassical atomfield interaction, the ringing for the SF at $420 \mathrm{~nm}$ is different than that for the SF at $5 \mu \mathrm{m}$. Neither diffraction nor transverse effects are included in the simulations. Therefore, the ringing in Fig. 9 cannot represent the observed ringing in Fig. 4. However the origins of the simulated ringing in Fig. 9 and observed ringing in Fig. 4 are consistent.

\section{CONCLUSION}

Rubidium atomic vapor was excited to the $5 D$ from the $5 S$ state via a two-photon process with 100 -fs laser pulses. The experiment was designed to measure the SF emission on the $6 P-5 S$ transition at $420 \mathrm{~nm}$. The spectrum of the $420 \mathrm{~nm}$ light was measured as a function of pulse energy. A simple correspondence between the $420 \mathrm{~nm}$ light intensity and input power was obtained. The temporal characteristics of the $420 \mathrm{~nm}$ SF was studied using an ultrafast streak camera.

Our system furnished an observation of a SF pulse lasting a few tens of picoseconds from a dense rubidium atomic vapor. On this time scale, relaxation processes such as spontaneous emission of the individual atoms, the Doppler effect and atomic coherence or collisional dephasing are negligible. The SF pulse shape changed considerably for different input pulse energies. For low power, significant temporal ringing was recorded. For high power, however, the ringing was not observed.

A numerical simulation, based on a semiclassical theory for five-level atoms with no free fitting parameters, yielded excellent agreement with our experimental data. Furthermore, the experimental results were compared with several existing $\mathrm{SF}$ theories. The distinct change in the scaling laws for the peak intensity and delay time versus input power is noticeable. The oscillatory SF theory, in which the sample length is larger than the cooperation length, explains the observed data most successfully.

We believe this study will be useful in various applications directly related to SF processes. For instance, the possibility of rapid inspection of quantum interference using the temporal characteristics of the coherent emissions of multiple atoms could be of great interest [30].

\section{ACKNOWLEDGMENT}

We wish to thank the referee for valuable comments. The authors also thank A. Lvovsky, A. Sokolov, D. Pestov, G. S. Agarwal, S. Harris, S. Mukamel, I. Gabitov, O. Kocharovskaya, V. Kocharovsky, B. Strycker, M. Springer, S. Das, and L. Keldysh for their fruitful discussions. This work was supported by the NSF Grant No. EEC-0540832 (MIRTHE ERC), Office of Naval Research (N00014-071-1084, N00014-08-1-0948, and N00014-09-1-0888), and Robert A. Welch Foundation (Award No. A-1261).
[1] R. H. Dicke, Phys. Rev. 93, 99 (1954).

[2] N. E. Rehler and J. H. Eberly, Phys. Rev. A 3, 1735 (1971).

[3] R. Bonifacio and L. Lugiato, Phys. Rev. A 11, 1507 (1975).
[4] N. Skribanowitz et al., Phys. Rev. Lett. 30, 309 (1973).

[5] J. Marek, J. Phys. B: At. Mol. Phys. 12, L229 (1979).

[6] F. T. Arecchi and E. Courtens, Phys. Rev. A 2, 1730 (1970). 
[7] J. C. MacGillivray and M. S. Feld, Phys. Rev. A 14, 1169 (1976).

[8] H. M. Gibbs, in Cooperative Effects in Matter and Radiation, edited by C. M. Bowden et al. (Plenum, New York, 1977), p. 61.

[9] R. Friedberg and S. R. Hartmann, Phys. Lett. A 37, 285 (1971); Phys. Rev. A 13, 495 (1976).

[10] D. C. Burnham and R. Y. Chiao, Phys. Rev. 188, 667 (1969).

[11] F. P. Mattar, H. M. Gibbs, S. L. McCall, and M. S. Feld, Phys. Rev. Lett. 46, 1123 (1981).

[12] D. J. Heinzen, J. E. Thomas, and M. S. Feld, Phys. Rev. Lett. 54, 677 (1985).

[13] M. Gross and S. Haroche, Phys. Rep. 93, 301 (1982).

[14] A. V. Andreev, V. I. Emel'yanov, and Yu. A. Il'inskii, Cooperative effects in Optics (Institute of Physics, Bristol, 1993); M. G. Benedict, A. M. Ermolaev, V. A. Malyshev, I. V. Sokolov, and E. D. Trifonov, Super-radiance Multiatomic Coherent Emission (Institute of Physics, Bristol, 1996).

[15] J. Okada et al., Opt. Commun. 26, 189 (1978).

[16] J. Okada, X. Lu, and S. R. Hartmann, Opt. Commun. 27, 321 (1978).

[17] J. H. Brownell et al., Phys. Rev. Lett. 75, 3265 (1995).

[18] A. I. Lvovsky, S. R. Hartmann, and F. Moshary, Phys. Rev. Lett. 82, 4420 (1999).

[19] A. I. Lvovsky, Ph.D. thesis, Columbia University, 1998.

[20] E. Paradis, B. Barrett, A. Kumarakrishnan, R. Zhang, and G. Raithel, Phys. Rev. A 77, 043419 (2008).
[21] The cell was constructed by David Sarkisyan at the Institute for Physical Research, NAS of Armenia, Ashtarak-2, Armenia.

[22] K. Ikeda et al., J. Phys. Soc. Jpn. 48, 1636 (1980).

[23] F. Haake, J. W. Haus, H. King, G. Schroder, and R. Glauber, Phys. Rev. A 23, 1322 (1981).

[24] R. Florian, L. C. Schwan, and D. Schmid, Phys. Rev. A 29, 2709 (1984); R. Bausch et al., Europhys. Lett. 10, 445 (1989); D. Jansen and A. Stahl, ibid. 18, 33 (1992); H. Steudel, Ann. Phys. (Leipzig) 503, 563 (1991).

[25] G. O. Ariunbold et al., "An observation of picosecond UV pulses produced by coherent scattering of ir femtosecond pulses in atomic rubidium vapor" (submitted).

[26] D. Polder, M. F. H. Schuurmans, and Q. H. F. Vrehen, Phys. Rev. A 19, 1192 (1979).

[27] R. Bonifacio et al. in Cooperative Effects in Matter and Radiation, edited by C. M. Bowden et al. (Plenum, New York, 1977), p. 193; R. Bonifacio et al., in Coherence and Quantum Optics IV, edited by L. Mandel and E. Wolf (Plenum, New York, 1978), p. 939.

[28] H. M. Gibbs et al., Phys. Rev. Lett. A 39, 547 (1977).

[29] A. Kumarakrishnan et al., J. Opt. Soc. Am. B 22, 1538 (2005); A. Kumarakrishnan and X. Han, Phys. Rev. A 58, 4153 (1998).

[30] G. O. Ariunbold, V. A. Sautenkov, and M. O. Scully, A Rapid Inspection of Atomic Interference using Superuorescent Picosecond Pulses, in Cleo Conference OSA Technical Digest Series (Optical Society of America, 2010), paper CMA4. 